\title{
Method to Evaluate Steering and Alignment Algorithms for Controlling Emittance Growth*
}

\author{
Chris Adolphsen and Tor Raubenheimer \\ Stanford Linear Accelerator Center, Stanford University, Stanford California 94309
}

\section{Abstract}

Future linear colliders will likely use sophisticated beambased alignment and/or steering algorithms to control the growth of the beam emittance in the linac. In this paper, a mathematical framework is presented which simplifies the evaluation of the effectiveness of these algorithms. As an application, a quad alignment algorithm that uses beam data taken with the nominal linac optics, and with a scaled optics, is cvaliated in terms of the dispersive emittance growth remaining after alignment.

\section{INTRODUCTION}

A major concern in the design of future linear colliders is the emittance growth in the linac. For example, dispersive and wakeficld generated growth occurs when the beam travels offaxis as a result of misalignments of the quads, beam position monitors (BPMs) and accelerator structures. Stecring and alignment algorithms have been devised to reduce these offsets and their effects [1-3]. The basic method of evaluating these algorithms is to simulate the linac beam transport including misalignments, and then to apply the correction scheme. This is repeated for many misalignment configurations to accurately gauge the effectiveness of the algorithm.

Here we present an analytical approach that makes it much easier to explore the 'parameter space' of an algorithm by eliminating the need to do simulations. This approaich is generally applicable in cases where the emittance growth depends quadraticly on the misalignments. Some examples in which it can be easily applied are dispersive growth where there is little filamentation, and wakefield growth where the orbit perturbations are smaller than the orbit offsets in the accelcrator structures.

A good way to describe the approach is to apply it to the problem of the dispersive emittance growth that results from misaligned quads and BPMs. We first define the problem, then formulate a quad alignment algorithm, and finally evaluate the growth from first order dispersion. Briefly, we exploit the fact that the computed misalignments are linearly related to the BPM measurements used in the fit. Since the emittance growth also depends linearly on the misalignments, the growth can be expressed in terms of the measurement errors.

\section{ALIGNMENT PROBLEM}

As a specific example, we treat the case of misalignments in a linac consisting of a FODO lattice with a BPM located in

* Work supported by Department of Energy contract DI:-AC03-76SF-00515. the bore of each quad. We assume that a single bunch of constant mean energy and constant er.ergy spread traverses the linac. We also assume a constant spacing, $L$, between quads, and a constant phase advance per FODO cell, $\mu$. These parameters define the periodic beta function: its maximum value is

$$
\beta_{\max }=\frac{2 L}{\sin (\mu)}[1+\sin (\mu / 2)] .
$$

We note that the emittance results derived with these assumptions can be simply related to the case of constant gradient acceleration with a $\sqrt{E}$ scaling of beta.

In computing the beam motion, we consider only the steering effect of the quad misalignments. The relation between the resulting beam trajectory and the BPM measurements is illustrated in Figure 1. Here, a quad is offset relative to an alignment axis by $x_{q}$, and the BPM located in the quad is offset relative to the quad center by $x_{b}$. Not represented in this figure is the BPM measurement error, which we denote by $x_{r}$. Including this error, the BPM measurement, $x_{w n}$, is related to the orbit offset, $x$, by

$$
x_{m}=x-x_{q}-x_{b}+x_{r} .
$$

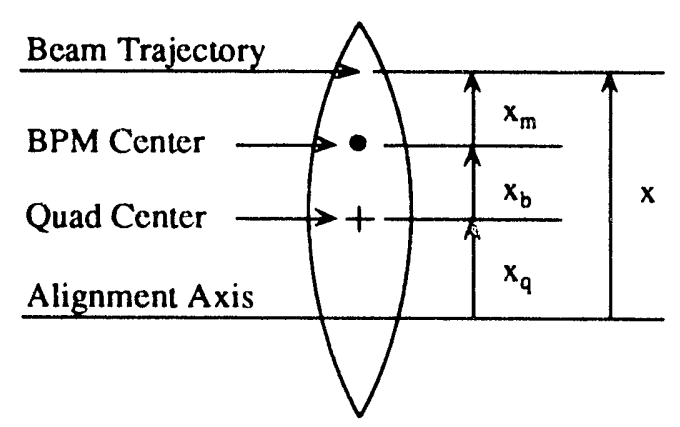

Figure 1. Illustration of a quad offsel, $x_{q}$, BPM offset, $x_{b}$, and BPM measurement $x_{m}$ for $x_{r}=0$.

From this relation, one can see that correcting the orbit by zeroing the BPMs (using stcering magnets for example) will result in an rms orbit offset at each quad that is the sum in quadrature of the rms BPM and quad offsets (assumed to be uncorrelated) and the BPM resolution. With this one-to-one steering approach, the resulting dispersion is proportional to the rms orbit offset. In an actual linac, the largest of the three contributions is likely to be from the quad offsets, followed by the BPM offsets. Hence, in a beam-based approach to reducing dispersion, it is reasonable to consider determining the quad offsets from the BPM data. The measurements from $\mathrm{N}$ contiguous BPMs can in fact be used to fit for the offsets of

\section{Presented at the Particle Accelerator Conference (PAC '93), Washington DC. May 17-20.1993 YB MASTER}


the $\mathrm{N}$ quads starting at the quad upstream of the first BPM. These offsets could then be used to correct the orbit by either moving the quads (assumed here for simplicity), or by equivalent adjustments to steering magnets.

One can also use BPM data taken with other lattice configurations to further reduce the quad offsets, or to allow for a fit for the BPM offsets as well. However, just minimizing the residual orbit offsets is not necessarily optimal for dispersion reduction since the correlations that arise between the offsets as a result of the fit affect how the dispersion source terms add. In fact, the results are often counterintuitive, making it all the more useful to have an analytic means of examining the problem.

\section{ALIGNMENT ALGORITHM}

To further our cxample, we next formulate a quad alignment algorithm based on two sets of BPM measurements, one taken with the nominal lattice, and one taken with a uniform scaling of the quad strengths. This scaling, whose size we denote by $\mathrm{Q} / \mathrm{Q}_{0}$, changes the phase advance per cell while maintaining periodic focusing. (We note that other types of lattice scaling are possible, and may be more efficient although less simple to implement.)

As a first step, we need to define an alignment axis to which the trajectory offsets are referenced. In practice, one is likely to align a group of $\mathrm{N}$ quads at a time, one group immediately after the next, so a good choice for the alignment axis is the line formed between the beam position at the first quad in the group, and the center of the first quad in the next group (...e., quad $\mathrm{N}+1$ ). This leaves the initial angle of the beam inconstrained, so it must also be included as a variable in the fit. We assume, however, that it does not change between the two sets of measurements.

As with any fitting procedure, we need to define a $\chi^{2}$ in torms of the variables and measurements involved, accounting for the errors and their correlations. The errors in this problem are the BPM offsets relative to the quads and the BPM measurement error, cach of which we assume to have a mean of $z$ cro and an rms of

$$
\sigma_{\mathrm{r}}^{2} \equiv\left\langle\mathrm{x}_{\mathrm{r}}^{2}\right\rangle \text { and } \sigma_{\mathrm{b}}^{2} \equiv\left\langle\mathrm{x}_{\mathrm{b}}^{2}\right\rangle
$$

for cach BPM. The resolution variable, $x_{r}$, differs from measurement-to-measurement while the offset variable, $x_{b}$, is common to all measurements. If we let ${ }^{1} \overrightarrow{\mathrm{x}}_{\mathrm{m}}$ and ${ }^{2} \overrightarrow{\mathrm{x}}_{\mathrm{m}}$ be vectors representing the two sets of $\mathrm{N}+1$ BPMs measurements (i.e., the $\mathrm{N}$ BPMs at the quads being aligned plus the next downstream BPM, with superscript 1 (2) denoting the measurements with the nominal (scaled) lattice), then we can write the full measurement vector and the corresponding error matrix as

$$
\overrightarrow{\mathrm{x}}_{\mathrm{m}} \equiv\left[\begin{array}{l}
{ }^{1} \overline{\mathrm{x}}_{\mathrm{m}} \\
{ }^{2} \overrightarrow{\mathrm{x}}_{\mathrm{m}}
\end{array}\right] \quad \Lambda \equiv\left\langle\overrightarrow{\mathrm{x}}_{\mathrm{m}} \overline{\mathrm{x}}_{\mathrm{m}}^{\prime}\right\rangle
$$

where the elements of $\Lambda$ are obtaincd from equations 2 and 3:

$$
\Lambda_{i, j}=\left[\sigma_{\mathrm{r}}^{2}+\sigma_{b}^{2}\right] \delta_{i, j}+\sigma_{b}^{2}\left(\delta_{i, j-N-1}+\delta_{i, j+N+1}\right) .
$$

The final ingredient for the fit is the functional dependence of the measurements on the fit variables. Fmm equation 2 , we need only an expression for $\mathrm{x}$ since $\mathrm{x}_{\mathrm{b}}$ and $\mathrm{x}_{\mathrm{r}}$ are treated as random variables. At quad $i$,

$$
x_{i}=R 12_{i, 1} \theta_{1}-\sum_{j=1}^{i-1} R 12_{i, j} K_{j} x_{q, j}
$$

and (for future reference)

$$
x_{i}^{\prime}=R_{22_{i, 1}} \theta_{1}-\sum_{j=1}^{i-1} R 22_{i, j} K_{j} x_{q, j}-\frac{K_{i}}{2} x_{q, i}
$$

where $R_{12_{i, j}}\left(R_{22_{i, j}}\right)$ is the $[1,2]([2,2])$ transport element between the center of quad $j$ and $i, K_{j}$ is the integrated quad strength, $\theta_{1}$ is the beam angle just upstream of the first quad, and $x_{q, i}$ is the offset of quad i (note $x_{q, N+1} \equiv 0$ ).

To simplify the algebra, we form a vector, $\vec{x}_{d}$, that is the difference of the BPM measurements and the fit function:

$$
\overrightarrow{\mathrm{x}}_{d} \equiv\left[\begin{array}{c}
{ }^{1} \ddot{x}_{d} \\
{ }^{2} \vec{x}_{d}
\end{array}\right] \equiv\left[\begin{array}{c}
{ }^{1} \vec{x}_{m}-\left({ }^{1} \bar{x}-\vec{x}_{q}\right) \\
{ }^{2} \vec{x}_{m}-\left({ }^{2} \vec{x}-\vec{x}_{q}\right)
\end{array}\right] .
$$

The expression for $\chi^{2}$ is then

$$
\chi^{2}=\vec{x}_{d}^{1} \Lambda^{-1} \vec{x}_{d}
$$

which when expanded yields

$$
\chi^{2} \propto \sum_{i=1}^{N+1}\left\{\left({ }^{1} x_{d, i}\right)^{2}+\left({ }^{2} x_{d, i}\right)^{2}-\frac{2 R^{2}}{1+R^{2}}\left({ }^{1} x_{d, i}\right)\left({ }^{2} x_{d, i}\right)\right\}
$$

where

$$
\mathrm{R} \equiv \sigma_{\mathrm{b}} / \sigma_{\mathrm{r}} .
$$

In the $\mathrm{R} \rightarrow 0$ limit, where the BPMs are perfectly aligned, $\chi^{2}$ reduces to the sum of squares of the two measurements, while in the $R \rightarrow \infty$ limit, it reduces to the sum of the measurement differences squared.

With this $\chi^{2}$, we next solve the set of equations generated when setting its derivative with respect to the fit variables to zero. Skipping this algebra, we note only that the resulting fit values are linearly related to the measurements. We express this by

$$
\overrightarrow{\mathbf{x}}_{\mathrm{v}} \equiv\left[\begin{array}{c}
\theta_{1} \\
\overrightarrow{\mathbf{x}}_{\mathrm{q}}
\end{array}\right] \equiv \mathbf{F} \overrightarrow{\mathbf{x}}_{\mathrm{m}}
$$

where $\mathbf{F}$ is a matrix which depends on $R$, and on the lattice parameters for the two measurements.

\section{DISPERSION}

With the alignment equations, we now compute the beam emittance growth due to the first order dispersion remaining after the alignment process. To begin, we evaluate the dispersion at the center of quad $\mathrm{N}$ (quad $\mathrm{N}+1$ is not used since the beam angle at this location is not constrained in the fit: this angle is corrected, however, during the alignment of the next 


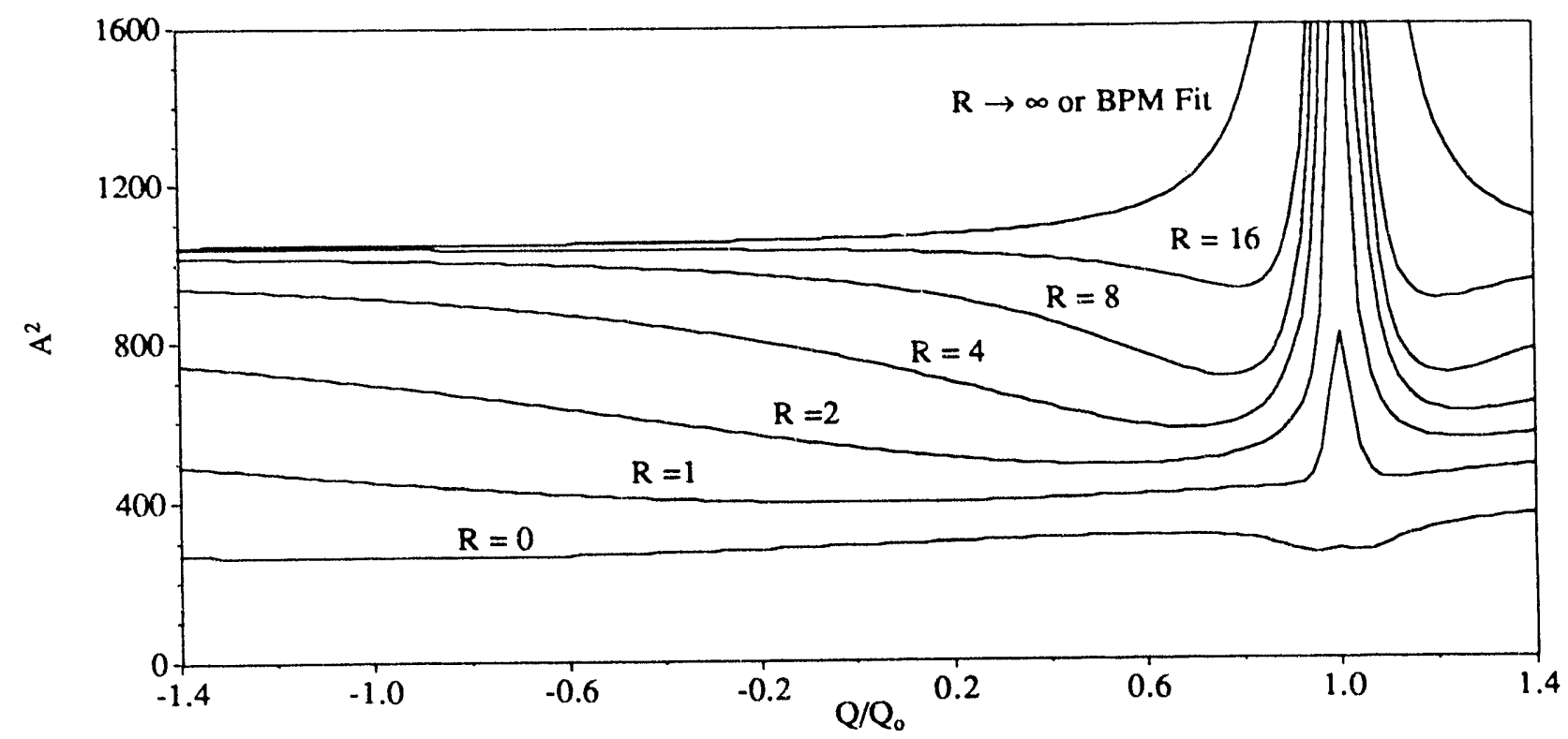

Figure 2. Scaled dispersion amplitude, $A^{2}$, as a function of $R$ and $Q / Q_{0}$ for $N=40$ and $\mu=90^{\circ}$. The $R \rightarrow \infty$ limit is equivalent to fitting for the BPM offsets.

group of $\mathrm{N}$ quads). Using equations 6 and 7 , the normalized dispersion vector can be written

$$
\vec{\eta}_{N}=\left[\begin{array}{cc}
\frac{1}{\sqrt{\beta_{N}}} & \frac{\partial^{1} x_{N}}{\partial \delta} \\
\sqrt{\beta_{N}} & \frac{\partial^{\prime} x_{N}^{\prime}}{\partial \delta}
\end{array}\right] \equiv D \vec{x}_{V}
$$

where $\mathbf{D}$ is a matrix that depends on the parameters of the nominal lattice, and $\delta$ is the fractional energy difference. To evaluate the emittance growth due to this dispersion, we average over the beam encrgy spread and over the quad alignment results. If $\varepsilon_{0}$ is the emittance of the beam entering the alignment region and $\sigma_{\delta}^{2}$ is the rms beam energy spread, the rms of the emittance at quad $N, \varepsilon_{N}$, is

$$
\frac{\left\langle\varepsilon_{N}^{2}\right\rangle^{1 / 2}}{\varepsilon_{0}}=\left(1+\frac{\sigma_{\delta}^{2}}{\varepsilon_{0}} \operatorname{Tr}\left[\left\langle\eta \eta^{l}\right\rangle\right]\right)^{1 / 2}
$$

where we ignore the effect of betatron chromaticity. Now we substitute the alignment results to compute the average:

$$
\begin{aligned}
& \operatorname{Tr}\left[\left\langle\eta \eta^{i}\right\rangle\right]=\operatorname{Tr}\left[\left\langle\mathbf{D} \overrightarrow{\mathbf{x}}_{\mathrm{v}} \overrightarrow{\mathbf{x}}_{\mathrm{v}}^{i} \mathbf{D}^{i}\right\rangle\right] \\
& =\operatorname{Tr}\left[\left\langle\mathbf{D F} \overrightarrow{\mathrm{x}}_{\mathrm{m}} \overrightarrow{\mathbf{x}}_{\mathrm{m}}^{\mathrm{l}} \mathbf{F}^{\mathrm{i}} \mathbf{D}^{\mathrm{i}}\right\rangle\right]=\operatorname{Tr}\left[\mathbf{D F} \wedge \mathbf{F}^{\mathrm{t}} \mathbf{D}^{\mathrm{l}}\right] .
\end{aligned}
$$

For convenience, we define a scaled dispersion amplitude as

$$
A^{2} \equiv \frac{\beta_{\max }}{\sigma_{r}^{2}} \operatorname{Tr}\left[\mathbf{D F} \wedge \mathbf{F}^{i} \mathbf{D}^{i}\right]
$$

which depends on $\mu, R, N$ and $Q / Q_{0}$. The emittance is then

$$
\frac{\left\langle\varepsilon_{N}^{2}\right\rangle^{1 / 2}}{\varepsilon_{0}}=\left(1+\frac{\sigma_{\delta}^{2} \sigma_{f}^{2}}{\beta_{\max } \varepsilon_{0}} A^{2}\right)^{1 / 2} .
$$

From a practical point of view, one wants $N$ to be large in order to reduce the dispersion from the uncorrected quad offsets at the ends of the alignment sections, but not too large that systematic effects, such as those duc to common errors in the quad strengths, are large. Based on experience with beambased alignment in the SLC, a reasonable value is $N=40$ for $\mu=90^{\circ}$. Figure 2 shows $A^{2}$ for this choice, and various values of $R$, as a function of $Q / Q_{0}$ over the range in which the phase advance per cell is $<180^{\circ}$.

For $Q / Q_{0}=1$, where two scts of the nominal lattice data are used, $A^{2}$ scales as $1+2 R^{2}$ since the effect of the BPM offsets and measurement errors are indistinguishable in the fit. Outside of the $Q / Q_{0}=1$ region, $A^{2}$ saturates as $R \rightarrow \infty$ since the difference of the orbits from the two lattices is independent of the BPM offsets, and hence independent of $R$. This is true even though the orbit offsets after alignment still scale as $\mathbf{R}$ for $R \gg 1$. Another interesting result is that if the BPM offsets are included as variables in the fit, the dispersion is the same as the $\mathrm{R} \rightarrow \infty$ case. Allowing the BPM offsets to vary apparently leaves the quad offsets sensitive to only the difference orbit. In this case, however, the orbit offsets after alignment do not depend on the initial value of $R$ (the error in determining the BPM offsets is about $1.3 \sigma_{r}$, independent of $N$ ). Finally, we note that $A^{2}$ is essentially linear with $N$ at $Q / Q_{0}=1$, and at values outside of the peak region. In the transition region, the widths of the peaks decrease as $\mathrm{N}$ increases.

\section{Y. REFERENCES}

11] C. Adolphsen et. al., SLAC-PUB-4902, (March 1989).

[2] T.O. Raubenheimer and R. Ruth, Nucl. Instr. and Meth. A302, 191 (1991).

[3] T.O. Raubenheimer, Nucl. Instr, and Mcth. A306, 61 (1991). 

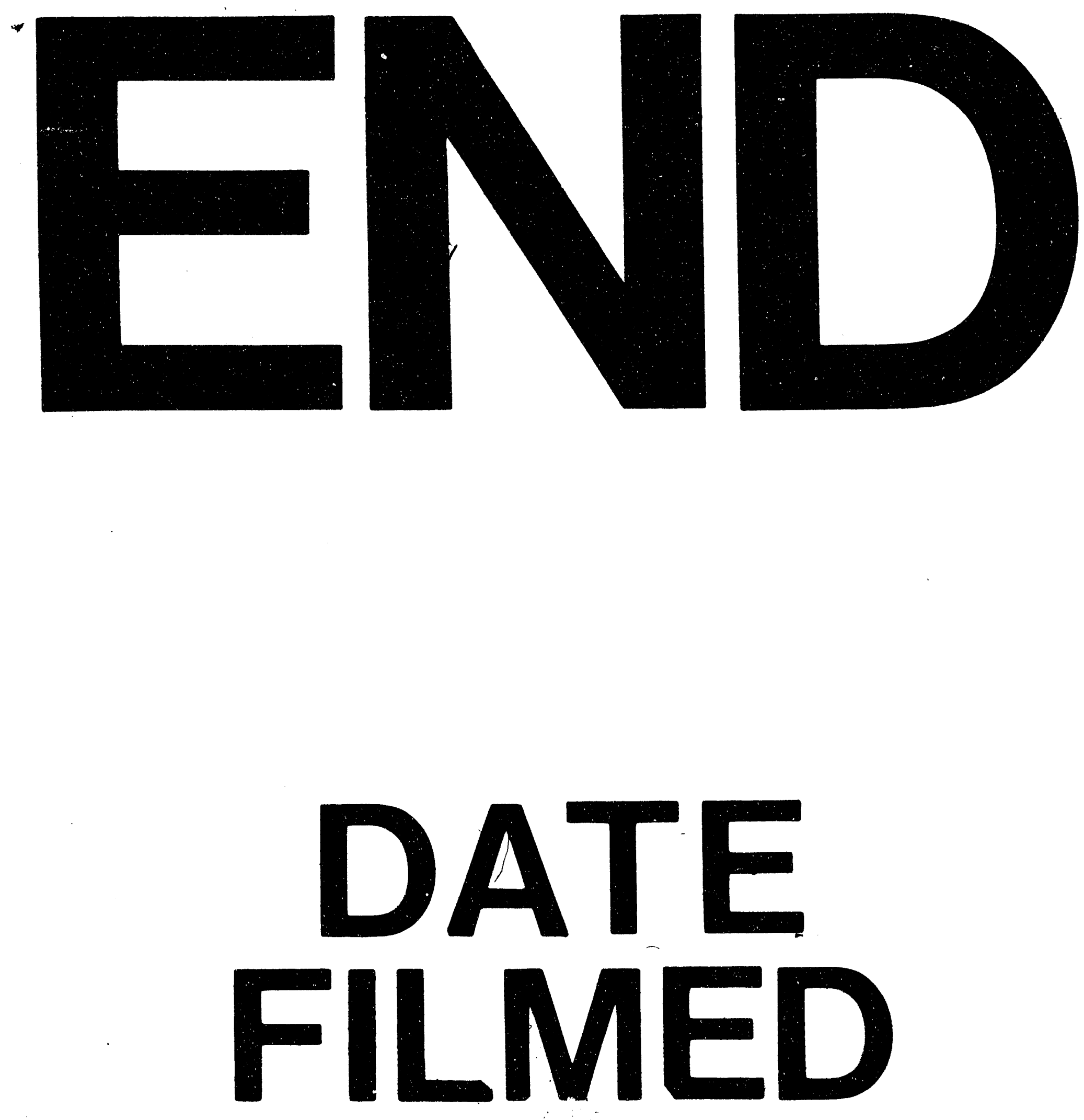

I

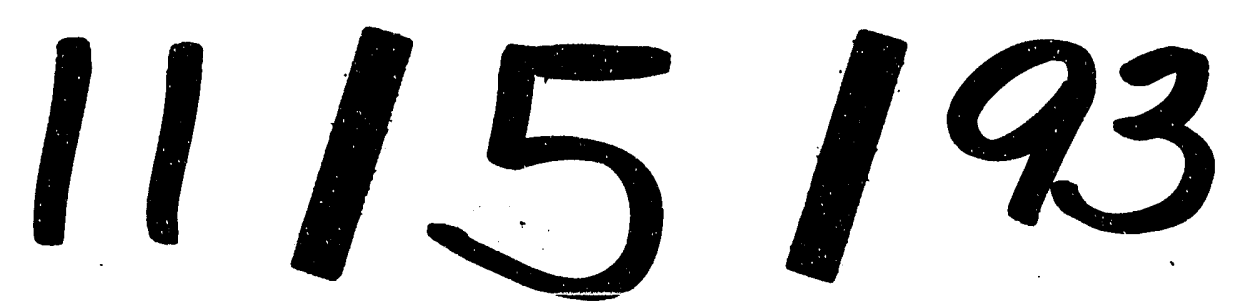


